\title{
The Relevant Inferences of Electrodynamics under Non-Void Backgrounds
}

\author{
An He \\ Chongqing University, Chongqing, China \\ Email: Hoane@163.com
}

How to cite this paper: He, A. (2019) The Relevant Inferences of Electrodynamics under Non-Void Backgrounds. Open Access Library Journal, 6: e5713.

https://doi.org/10.4236/oalib.1105713

Received: August 16, 2019

Accepted: September 16, 2019

Published: September 19, 2019

Copyright ( 2019 by author(s) and Open Access Library Inc.

This work is licensed under the Creative Commons Attribution International License (CC BY 4.0).

http://creativecommons.org/licenses/by/4.0/

(c) () Open Access

\begin{abstract}
The paper was based on the definition of time-rate, and founded the general transformation between reference systems that background had different standard of time, and the self-consistent theoretical system has been formed to promote the classical electrodynamics. According to the principle, more discussion about the relationship between structure of time-rate in space and physical properties in its own area (gravity, centrifugal force, acceleration system) was presented. In view of the above, the paper expounded the role of macro mechanism of gravity and inertia force, and unified them finally. The possibility of tremendous instantaneous acceleration has been put forward.
\end{abstract}

\section{Subject Areas}

Special Theory of Relativity, Theoretical Physics

\section{Keywords}

Lorentz Transformation, Field Reference System, Time-Rate, Gravitation, Inertial Force

\section{Introduction}

The achievements of the relativistic views on the space-time make us known that the concept of the universal time can't describe the complexity of the space-time properties availably. On the contrary, the fact that velocity of time-passing is not same either in different position of gravitation field, suggests that the region of space which has different time feature is objective existence; it should be the basic property for the space-time also and must influence the physical process because of the mutual action between matter and field. So, there is more widespread meaning to describe the property of space-time in different background conditions.

The existence of the gravitational field damages the uniformity of physical 
properties of the space, including the time features; it means that the physical properties of the space are different in the real physical environment. The widespread fact makes us believe that describing the space-time relations under the conditions of different backgrounds and its impact on the physical process of objectively should have more general significance. However, the background of the coordinate system usually defaults to the same; as an implied condition in the Lorentz transformation, it will no doubt make the theory of the scope of application limited by certain.

\section{Definitions and Basic Properties of Field Reference System and Time-Rate}

In the theories that with the argumentation of field, any physical process has to depend on the existence of the space-time background, but its properties of reference system and time-scale are always implicit and consistent. Since the space-time is the basic attributes of the realistic physical environment, and it couldn't be ignored for the motion of substance, the reference system and its properties of background should be regarded whole to participated in the description for physical process together, It would influence our research to the area whose feature of time-passing distinguishes to each other in the cosmos by all means. So we call the coordinates whose background has given time properties the "Field reference system" from this angle. There is reason to believe, the same laws will be valid for all frames of field reference which the equations of mechanics hold good. At the same time, in order to describe time feature of any reference system, we defined that within per unit time of the eigen system clock, the elapsed time of system which respect to the Eigen reference system was the velocity of time-passing, hereinafter referred to as "Time-rate".

Take standard reference systems of the special relativity as $S$ and $S^{\prime}$ with the same properties at all directions and independent mutually, we establish time-rate that system $S^{\prime}$ relative to system $S$ is $\mathscr{F}$, In order to show the difference of the background between the reference systems, we use (Figure 1). According to the definition of time-rate we have

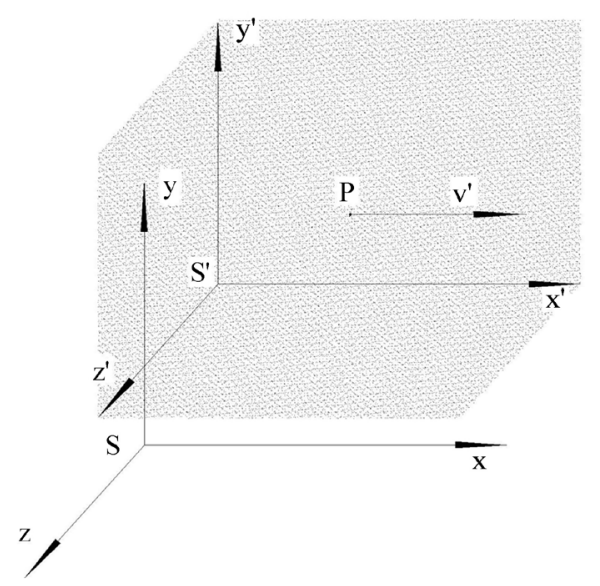

Figure 1. Field reference systems with different time feature. 


$$
\mathscr{F}=t^{\prime} / t
$$

Similarly, time-rate that system $S$ relative to $S^{\prime}$ is $\mathscr{F}^{\prime}=t / t^{\prime}$. On the other hand, because the relativity of simultaneous result in length constringency, so there is the transformation of length between the field reference systems $S$ and $S^{\prime}$ are relatively static.

$$
r=r^{\prime}
$$

Equation (1) and Equation (2) which mentioned above were constituted the basic transformations between the static field reference systems.

\subsection{Description for the Velocity of the Same Moving Body between Field Reference Systems}

If system $S^{\prime}$ and $S$ are superposed and relatively static, at the time $t=t^{\prime}=0$, a particle $P$ moves along the forward direction of $\mathrm{x}$-axis from origin of coordinates. When viewed from the systems each, we can get speed relation with Equation (1) and Equation (2)

$$
v=\frac{\mathrm{d} r}{\mathrm{~d} \tau}=v^{\prime} \mathscr{F}
$$

Though the system $S$ and $S^{\prime}$ to be relatively static, but they ordered the velocity of particle $P$ for different results. So, when we make different descriptions of velocity for the same one moving body from different field reference systems, it couldn't indicate that there had relative motion between them.

\subsection{Velocity of Relative Motion between the Field Reference Systems}

When the system $S^{\prime}$ moving with velocity $v$ relative to $S$ in the forward direction of the public axis $x-x^{\prime}$. When we viewed from system $S$, there has relation $v=v^{\prime} \mathscr{F}$, for the same reason, viewed from the $S^{\prime}$, then we have $v^{\prime}=-v / \mathscr{F}$. It is obvious that $|v| \neq\left|v^{\prime}\right|$. So when we viewed from the reference systems each, the velocity of relative motion are opposite and unlikeness.

Thus, because the Field reference systems had different Time-rates, we might make different results of measuring for the same case with its standard of time and length, even if the field references in the state at relatively static. It's different from our previous experience. This indicated that the equality of relative motion would be valid no longer, and it makes us know that the velocity of photon must be dependent on time-rate of the Field reference system, therefore we modify the parameters as follows, the velocity of light in vacuum is marked $c_{0}$. And when we replace the particle $P$ by the ray, the transformation of velocity of light would be expressed as

$$
c=c^{\prime} \mathscr{F}=c^{\prime} / \mathscr{F}^{\prime}
$$

Hence, we can try to found the current transformations between the Field reference systems, and extend the classical electrodynamics to any coordinates, which background with properties of any time scale of the field reference system 
finally.

\section{Transformations between Inertial Field Reference Systems}

Let's take two standard systems as $S$ and $S^{\prime}$ of the special relativity, and set its time-rate is 1 and $\mathscr{F}$ respectively, then we have the kinematics relations [1]: $x^{\prime}=a_{11} x+a_{14} t, \quad y^{\prime}=y, \quad z^{\prime}=z, t^{\prime}=a_{41} x+a_{44} t$. We recorded the coordinates of the ray were $(x, t)$ and $\left(x^{\prime}, t^{\prime}\right)$ respectively from the references each, so, $x^{\prime}=c^{\prime} t^{\prime}, x=c t$. According to the kinematics, we get formulas as follows:

$$
\begin{gathered}
x^{\prime}=a_{11}(x-v t) \\
t^{\prime}=a_{11}\left(t-v x / c^{2}\right) \mathscr{F}
\end{gathered}
$$

It is to be noted that the equality of relative motion would be valid no longer between the Field reference systems because of time-rate, therefore we might found the converse transformation with the method above and have the opposite Equations of (7) and (8)

$$
\begin{gathered}
x=a_{11}\left(x^{\prime}+v^{\prime} t^{\prime}\right) \\
t=a_{11} \frac{1}{\mathscr{F}}\left(t^{\prime}+v^{\prime} x^{\prime} / c^{\prime 2}\right)
\end{gathered}
$$

We can calculate the values with Equations (5)-(8) together now. In fact, if we keep the interval of four-dimensional constant, namely $S_{12}^{2}=S_{12}^{\prime 2}$, Substituting the Lorenz transformation and the Equation (1) into it, at the same time, the velocity of light was remains the same no longer in the field reference system conditions, and it should be followed the relation (4), so we can get the new transformations which in the condition of field reference system and are expressed with matrix as follow:

I) $\left[a_{\mu \nu}\right]=\left[\begin{array}{cccc}\gamma & 0 & 0 & i \beta \gamma \\ 0 & 1 & 0 & 0 \\ 0 & 0 & 1 & 0 \\ -i \beta \gamma \mathscr{F} & 0 & 0 & \gamma \mathscr{F}\end{array}\right]$

II) $\left[a_{\mu v^{\prime}}^{\prime}\right]=\left[\begin{array}{cccc}\gamma^{\prime} & 0 & 0 & -i \beta^{\prime} \gamma^{\prime} \\ 0 & 1 & 0 & 0 \\ 0 & 0 & 1 & 0 \\ i \beta^{\prime} \gamma^{\prime} \mathscr{F}^{\prime} & 0 & 0 & \gamma^{\prime} \mathscr{F}^{\prime}\end{array}\right]$

where $\gamma=\frac{1}{\sqrt{1-\beta^{2}}}=\frac{1}{\sqrt{1-\beta^{\prime 2}}}=\gamma^{\prime}, \quad \beta=\frac{v^{2}}{c^{2}}=\frac{v^{\prime 2}}{c^{\prime 2}}=\beta^{\prime}$

\section{Mass-Energy Transformation between the Field Reference Systems}

First, we marked Static mass and kinetic mass with $m_{0}^{\prime}$ and $m^{\prime}$ respectively in system $S^{\prime}$, and defined $m_{0}$ and $m$ alike in system $S$. Take a case of symmetrical collision in system $S$, the initial speed of object $A$ and $B$ are equal and opposite. Namely, $u_{y A}^{\prime}=-u_{y B}^{\prime}, u_{x A}^{\prime}=-u_{x B}^{\prime}$, The system $S^{\prime}$ moving with the velocity $v$ relative 
to system $S$ in the forward direction of the $x^{\prime}$-axis, according to the request for the transformations of velocity, we have

$$
u_{x A}^{\prime}=-u_{x B}^{\prime}=-\frac{v}{\mathscr{F}}
$$

So $v=\frac{c^{2}}{u_{x B}}\left(1-\sqrt{1-u_{x B}^{2} / c^{2}}\right)$.

Substituting it into the conservation of momentum relation, then

$$
m_{B}=\frac{1}{\sqrt{1-u_{x B}^{2} / c^{2}}} m_{A}
$$

Applied the result to towards wiping collision, the object $A$ and $B$ without the component of velocity along the y-axis, so the object $A$ in the state at rest in system $S$ and the Equation above turned to the mass ratio of the kinetic mass and Static mass in the Field reference system now. Substituting transformations of velocity into Eq. above, and we have

$$
m=\frac{1}{\gamma^{\prime}}\left(1+v^{\prime} u_{x}^{\prime} / c_{0}^{2}\right) \frac{m_{0}}{\sqrt{1-u^{\prime 2} / c_{0}^{2}}}
$$

Let's take another special case of towards wiping collision, that the object $A$ and $B$ without the component of momentum along the y-axis and $z$-axis. So the component of momentum should be equal in those directions for the system $S$ and $S^{\prime}$, hence

$$
m u_{y}=m \frac{u_{y}^{\prime} \sqrt{1-v^{\prime 2} / c_{0}^{2}}}{1+v^{\prime} u_{x}^{\prime} / c_{0}^{2}} \mathscr{F}=m^{\prime} u_{y}^{\prime}
$$

Here, according to the special relativity

$$
m^{\prime}=\frac{m_{0}^{\prime}}{\sqrt{1-\left(u_{x}^{\prime 2}+u_{y}^{\prime 2}+u_{z}^{\prime 2}\right) / c_{0}^{2}}}
$$

So there is the integrated transformation of mass between Field reference systems

$$
m=\frac{1}{\gamma^{\prime}}\left(1+v^{\prime} u_{x}^{\prime} / c_{0}^{2}\right) \frac{m_{0}^{\prime}}{\sqrt{1-u^{\prime 2} / c_{0}^{2}} \mathscr{F}}
$$

Compared the Equation (10) with Equation (9), and have

$$
m_{0}=m_{0}^{\prime} / \mathscr{F}
$$

From the formula (10), when $\mathscr{F}=1, v^{\prime}=0$, then $u=u^{\prime}, m_{0}=m_{0}^{\prime}$, and we have the Equation $m=m_{0} / \sqrt{1-u^{2} / c_{0}^{2}}$ in special relativity. So the kinetic energy is expressed as below which in the Field reference system conditions.

$$
E_{k}=m_{0} c_{0}^{2}\left(\frac{1}{\sqrt{1-v^{2} / c_{0}^{2}}}-1\right)
$$

Let Equation (10) have dimension of energy and keep its simplest form, then can multiply the value $c_{0}^{2}$ at both sides of the Eq., hence 


$$
E=\frac{1}{\gamma^{\prime} \mathscr{F}}\left(E^{\prime}+P_{x}^{\prime} v^{\prime}\right)
$$

when $u^{\prime}=0, v^{\prime}=0$, thus $m^{\prime}=m_{0}^{\prime}, m=m_{0}$, and then we have transformation of energy between field reference systems are relatively static.

$$
E=E^{\prime} / \mathscr{F}
$$

\section{Transformation of Force}

According to the classic definition, the component of forces in the $x$-axis is expressed as

$$
F_{x}=\frac{\mathrm{d} P_{x}}{\mathrm{~d} t}=\left(F_{x}^{\prime}+\frac{v^{\prime}}{c_{0}^{2}} \frac{\mathrm{d} E^{\prime}}{\mathrm{d} t^{\prime}}\right) \frac{\mathscr{F}}{1+v^{\prime} u_{x}^{\prime} / c_{0}^{2}}
$$

As $\frac{\mathrm{d} E^{\prime}}{\mathrm{d} t^{\prime}}=F^{\prime} u^{\prime}=F_{x}^{\prime} u_{x}^{\prime}+F_{y}^{\prime} u_{x}^{\prime}+F_{z}^{\prime} u_{z}^{\prime}$, substituting it into Equation (15), then

$$
F_{x}=\left[F_{x}^{\prime}+\frac{v^{\prime}}{c_{0}^{2}+u_{x}^{\prime} v^{\prime}}\left(F_{y}^{\prime} u_{y}^{\prime}+F_{z}^{\prime} u_{z}^{\prime}\right)\right] \mathscr{F}
$$

Transformations of force in the direction of $y$-axis and $z$-axis are expressed as follows

$$
\begin{aligned}
& F_{y}=\frac{F_{y}^{\prime} \gamma^{\prime}}{\left(1+v^{\prime} u_{x}^{\prime} / c_{0}^{2}\right)} \mathscr{F} \\
& F_{z}=\frac{F_{z}^{\prime} \gamma^{\prime}}{\left(1+v^{\prime} u_{x}^{\prime} / c_{0}^{2}\right)} \mathscr{F}
\end{aligned}
$$

And we can get the converse transformations by the same way

$$
\begin{gathered}
F_{x}^{\prime}=\left[F_{x}-\frac{v}{c^{2}-u_{x} v}\left(F_{y} u_{y}+F_{z} u_{z}\right)\right] \frac{1}{\mathscr{F}} \\
F_{y}^{\prime}=\frac{F_{y} \gamma}{\left(1-v u_{x} / c^{2}\right) \mathscr{F}} \\
F_{z}^{\prime}=\frac{F_{z} \gamma}{\left(1-v u_{x} / c^{2}\right) \mathscr{F}}
\end{gathered}
$$

\section{Transformations of the Electromagnetic Field}

The Lorentz force can be expressed as follows
III) $\left\{\begin{array}{l}F_{x}=q_{0}\left(E_{x}+u_{y} B_{z}-u_{z} B_{y}\right) \\ F_{y}=q_{0}\left(E_{y}+u_{z} B_{x}-u_{x} B_{z}\right) \\ F_{z}=q_{0}\left(E_{z}+u_{x} B_{y}-u_{y} B_{x}\right)\end{array}\right.$
IV) $\left\{\begin{array}{l}F_{x}^{\prime}=q_{0}\left(E_{x}^{\prime}+u_{y}^{\prime} B_{z}^{\prime}-u_{z}^{\prime} B_{y}^{\prime}\right) \\ F_{y}^{\prime}=q_{0}\left(E_{y}^{\prime}+u_{z}^{\prime} B_{x}^{\prime}-u_{x}^{\prime} B_{z}^{\prime}\right) \\ F_{z}^{\prime}=q_{0}\left(E_{z}^{\prime}+u_{x}^{\prime} B_{y}^{\prime}-u_{y}^{\prime} B_{x}^{\prime}\right)\end{array}\right.$

According to the transformation of force, we insert the second Equation of (III) 
into Equation (20)

$$
F_{y}^{\prime}=\frac{q_{0}\left(E_{y}+u_{z} B_{x}-u_{x} B_{z}\right)}{\gamma\left(1-v u_{x} / c^{2}\right) \mathscr{F}}
$$

Substituting transformations of velocity into to the Equation above, and compared it with Equations of the Lorentz force, then we have

$$
F_{y}^{\prime}=q_{0}\left[\frac{1}{\sqrt{1-v^{\prime 2} / c_{0}^{2}}}\left(\frac{E_{y}}{\mathscr{F}}-v^{\prime} B_{z}\right)+u_{z}^{\prime} B_{x}-\frac{u_{x}^{\prime}}{\sqrt{1-v^{\prime 2} / c_{0}^{2}}}\left(B_{z}-\frac{E_{y} v^{\prime}}{c_{0}^{2} \mathscr{F}}\right)\right]
$$

And insert the third Equation of (III) into (21) by the same way, we have

$$
F_{z}^{\prime}=q_{0}\left[\gamma\left(\frac{E_{z}}{\mathscr{F}}+v^{\prime} B_{y}\right)+u_{x}^{\prime} \gamma\left(B_{y}+\frac{E_{z} v^{\prime}}{c_{0}^{2} \mathscr{F}}\right)-u_{y}^{\prime} B_{x}\right]
$$

when $u_{y}=u_{z}=u_{y}^{\prime}=u_{z}^{\prime}=0$, then the first Equation of (III) and (IV) gives us

$$
\left\{\begin{array}{l}
F_{x}=q_{0} E_{x} \\
F_{x}^{\prime}=q_{0} E_{x}^{\prime}
\end{array}\right.
$$

Then according to the transformation of force, we obtain: $F_{x}=F_{x}^{\prime} \mathscr{F}$

So

$$
E_{x}^{\prime}=E_{x} / \mathscr{F}
$$

Compared Equation (22), Equation (23) with 2nd and 3rd Equations of (IV) respectively, we have transformations of electromagnetic field as follows

V) $\left\{\begin{array}{l}E_{x}^{\prime}=\frac{E_{x}}{\mathscr{F}} \\ E_{y}^{\prime}=\gamma\left(E_{y}-v B_{z}\right) \frac{1}{\mathscr{F}} \\ E_{z}^{\prime}=\gamma\left(E_{z}+v B_{y}\right) \frac{1}{\mathscr{F}}\end{array}\right.$

VI) $\left\{\begin{array}{l}B_{x}^{\prime}=B_{x} \\ B_{y}^{\prime}=\gamma\left(B_{y}+E_{z} v / c^{2}\right) \\ B_{z}^{\prime}=\gamma\left(B_{z}-E_{y} v / c^{2}\right)\end{array}\right.$

In a similar way, there are the converse transformations of the electromagnetic field

$$
\begin{aligned}
& \text { VII })\left\{\begin{array}{l}
E_{x}=E_{x}^{\prime} \mathscr{F} \\
E_{y}=\gamma^{\prime}\left(E_{y}^{\prime}+v^{\prime} B_{z}^{\prime}\right) \mathscr{F} \\
E_{z}=\gamma^{\prime}\left(E_{z}^{\prime}-v^{\prime} B_{y}^{\prime}\right) \mathscr{F}
\end{array}\right. \\
& \text { VIII) }\left\{\begin{array}{l}
B_{x}=B_{x}^{\prime} \\
B_{y}=\gamma^{\prime}\left(B_{y}^{\prime}-v^{\prime} E_{z}^{\prime} / c_{0}^{2}\right) \\
B_{z}=\gamma^{\prime}\left(B_{z}^{\prime}+v^{\prime} E_{y}^{\prime} / c_{0}^{2}\right)
\end{array}\right.
\end{aligned}
$$

We assume a plate capacitor remain its state at rest and horizontal along the 
direction of x-axis in system $S^{\prime}$, so $E_{x}^{\prime}=E_{z}^{\prime}=B^{\prime}=0, E_{y}^{\prime}=E$. Then we can get relations of the electric field and the magnetic field with Equation (VII) and (VIII) in system $S^{\prime}, E=\gamma^{\prime} E_{y}^{\prime} \mathscr{F}, B=v^{\prime} \gamma^{\prime} E_{y}^{\prime} / c_{0}^{2}$. From the relation of energy density, then

$$
W=\frac{1}{2} \gamma^{\prime 2} E^{\prime 2}\left(\varepsilon \mathscr{F}^{2}+v^{\prime 2} / \mu c_{0}^{4}\right)
$$

We assume $c=1 / \sqrt{\varepsilon \mu}$, thus $\varepsilon=1 / \mu c^{2}=1 / \mu c_{0}^{2} \mathscr{F}^{2}$, insert the Equation into (25), then

$$
W=\frac{1}{2} \gamma^{\prime 2} E^{\prime 2} \varepsilon\left(1+v^{\prime 2} / c_{0}^{2}\right) \mathscr{F}^{2}
$$

when $v^{\prime}=0$, the Equation above has been simplified to $W=\frac{1}{2} \varepsilon E^{\prime 2} \mathscr{F}^{2}$. According to the transformation of energy, we compared the Eq. with it and have $\varepsilon=\frac{\varepsilon_{0}}{\mathscr{F}^{3}}, \mu=\mu_{0} \mathscr{F}$.

So the transformation of conductivity and magnetic permeability is the basic reason for the changed of velocity of light in field reference system.

This shows that when time-rate be equal to 1 , the theories returned to the category of special relativity completely. And we can see from the result that taking the concept of time-rate into Lorentz transformation is reasonable. So based on this study, we can analyze the structural form and physical feature of Time-rate in gravitation with the principle.

\section{Physical Meaning of Time-Rate}

\subsection{Time-Rate of Moving Reference System}

We could describe the feature of time-passing for any systems with time-rate. The time delays effect determined time-rate of systems at motion which is without effect of gravitation and any other field. So there is the relation of time between motorial reference system $S^{\prime}$ and $S$ :

$$
t=\frac{t^{\prime}}{\sqrt{1-v^{2} / c_{0}^{2}}}
$$

According to the definition, time-rate of the reference system $S^{\prime}$ relative to the system $S$ should be:

$$
\mathscr{F}=\frac{\mathrm{d} t^{\prime}}{\mathrm{d} t}=\sqrt{1-v^{2} / c_{0}^{2}}
$$

Therefore, the clock in the system $S^{\prime}$ would be slower than $S$.

Because of the relativity of the motion, there has the same form either when viewed from the reference system $S$. So we affirmed time-rate was determined by Equation (27) between the systems at motion with the velocity $v$ relative to one another in space without acts by any field. 


\subsection{Time-Rate of the Gravitation Field}

Let's assumed a case of conversion between the kinetic energy and potential energy in gravity field, and rewrite the form of the kinetic energy for relativity, then have:

$$
m_{0} c_{0}^{2}\left(\frac{1}{\sqrt{1-v^{2} / c_{0}^{2}}}-1\right)=G \frac{M m_{0}}{r}
$$

Tidy the Equation above and compared it with Equation (27), then:

$$
\mathscr{F}=\sqrt{1-2 G M / r c_{0}^{2}}
$$

This Equation was included the gravitational feature. As a relation of time-rate, it might be related with the gravitation, and it expresses time-rate in the place with distance $r$ from the center of the $M$. So time-rate of gravitation field is the function of mass and distance, it reflects the fact that the velocity of time-passing is disaffinity for different position in gravitation.

Assume time-rate at sea level and at an altitude $\mathrm{H}$ are $\mathscr{F}_{0}$ and $\mathscr{F}_{H}$ respectively. The elapse time of them ware $\tau_{0}=\tau \mathscr{F}_{0}$ and $\tau_{H}=\tau \mathscr{F}_{H}$ within unit time of the eigen system, and time-rate difference is $\Delta \tau=\tau_{H}-\tau=\tau\left(\mathscr{F}_{H}-\mathscr{F}_{0}\right)$, unfurl the formula with binomial theorem and we have:

$$
\Delta \tau=G M / c_{0}^{2}\left(1 / r_{0}-1 / r_{H}\right) \text {. }
$$

According to the formula, we can calculate the time delay between any height in the gravitational field.

See from the Equation (28), time-rate increases with the distance increasing from the barycenter. The nearer to barycenter, the stronger the gravitation is, and the slower the time-passing is too. So a kind of gradient structure of time-rate which was changed gradually would be formed around the mass in space. There is reason to believe that the structural form of time-rate has close contact with the physical properties of gravitation field.

In order to vividly describe the structure characteristics and distribution of time-rate in the gravitational field, we adopt the way of graphics, the density of dots to represent the size of time-rate. The stronger the gravity, the greater the density of points is, and the smaller time-rate is too. So time-rate of space in the gravitational field is formed as shown in Figure 2.

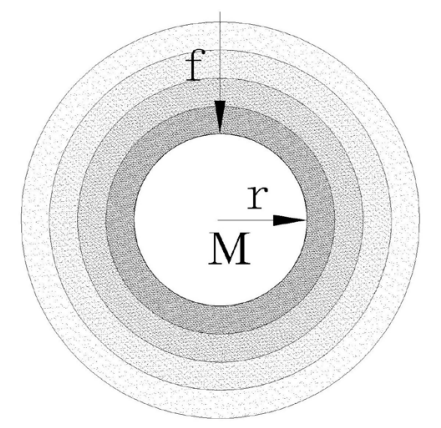

Figure 2. Structural of time-rate in gravitational field. 


\section{Universal Gravitation}

Let's take mass $m_{0}$ with distance $r$ from the center in gravitation field of the mass $M$. When viewed from the eigen system, the energy in its place of gravitation is $E$, according to the Equation (14) and evaluate the gradient with distance:

$$
\frac{\mathrm{d} E}{\mathrm{~d} r}=m_{0} c_{0}^{2} \frac{\mathrm{d} \mathscr{F}^{-1}}{\mathrm{~d} r}=-\frac{1}{\mathscr{F}^{3}} G \frac{M m_{0}}{r^{2}}
$$

According to classical mechanics, this Equation has dimension of force and with the negative values, it shows that it's a kind of centripetal force. It makes us thought of the universal gravitation very naturally. Change the form of the Equation and have:

$$
U=-\frac{1}{\mathscr{F}^{3}} G \frac{M m_{0}}{r}=-G \frac{M m_{0}}{r}-\frac{3}{2} \frac{v^{2}}{c_{0}^{2}} \frac{G M m_{0}}{r}+\cdots
$$

where: $v^{2}=\frac{2 G M}{r}$.

Obviously, it is just the equation of gravitation in general relativity that includes the post Newtonian approximation. And it shows that Equation (29) is exactly the expression of universal gravitation which modified behind.

So the attraction between $M$ and $m_{0}$ should be:

$$
F_{M}=\frac{1}{{\sqrt{1-2 G M / r c_{0}^{2}}}^{3}} G \frac{M m_{0}}{r^{2}}, \quad F_{m_{0}}=\frac{1}{{\sqrt{1-2 G m_{0} / r c_{0}^{2}}}^{3}} G \frac{M m_{0}}{r^{2}}
$$

It is obvious that: $F_{M}>F_{m_{0}}$.

Here, the universal gravitation without the characteristics of force and recoil force, on the contrary, the attraction for the body may depend on time-rate gradient in the space that around headstream of gravitation. It also avoids the problem of the gravity action at distance.

So we can get the potential energy of $m_{0}$ in the gravitation of mass $M$, then:

$$
\Delta E_{\varphi}=\int_{u=0}^{u=u} F_{M} \mathrm{~d} r=m_{0} c_{0}^{2}\left(\frac{1}{\sqrt{1-2 G M / r c_{0}^{2}}}-1\right)
$$

The Equation above was opposite with the Equation (12) for kinetic energy of special relativity, that's Einstein's equivalence principle.

\section{Centrifugal Force}

We can see from the corresponding relations of the kinetic energy and potential energy in the relativistic category that, the field potential of the motorial system relative to the eigen system was identical effects with the corresponding partial area of the uniform gravitation. It's just the expression of Einstein's equivalence principle in inertial category.

This might extend the principle of force by the field hopefully. In rotating system, the farther away from the center of circle, the greater the velocity of the tangential movement is, and the smaller time-rate which respected to the center of circle or eigen system is. So it forms the structure that time-rate reduce gradually with the radii enlarge in the rotating system (Figure 3 ). 


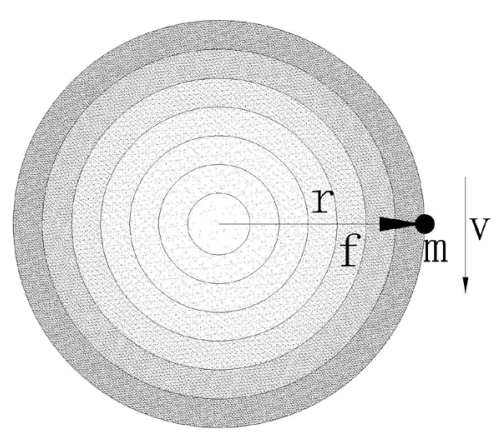

Figure 3. Structural of time-rate in centrifugal system.

Take an object with Static mass $m_{0}$ and distance $r$ form the centre of circle in the rotating system. It moves around the centre of circle with the angular velocity $\omega$, when viewed from the eigen system and Evaluate the gradient of distance:

$$
F_{\Phi}=\frac{\mathrm{d} E_{\Phi}}{\mathrm{d} r}=m_{0} c_{0}^{2} \frac{\mathrm{d}\left(1-\omega^{2} r^{2} / c_{0}^{2}\right)^{-1 / 2}}{\mathrm{~d} r}=\frac{1}{\mathscr{F}^{3}} m_{0} \frac{v^{2}}{r}
$$

It expressed that the direction of this force was outwardly with its positive values. It is to say that we get the centrifugal force in this way. Thus, the gradient of energy which caused by time-rate gradient change in space was the essential cause of gravitation that around the mass and the centrifugal force in rotating system. When time-rate keep the uneven distribution in space, and form a sort of gradient structure that time-rate gradual change with specific way, the mass or energy must be acted upon by force in its space for this, and the force pointed the direction where time-rate decreased.

\section{Inertial Force}

The relativity points out explicitly that the velocity of time-passing was different along the direction of acceleration at different position in the system with variable speed. As Professor Ta-You Wu stated in his book that "In Non-Inertial System, the measurements of time and space would be different everywhere" [2]. So, in interior region of the acceleration system, it forms the structure of gradual change that time-rate increase gradually at the direction of acceleration, and there must conduced a sense of force inside the system in the opposite direction with the principle above, which is just the inertial force that object was acted upon in motorial system with variable speed (Figure 4).

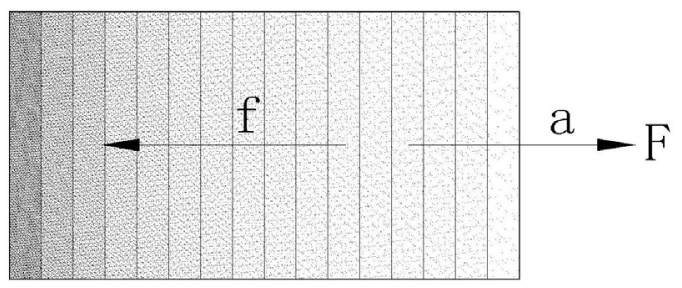

Figure 4. Structural of time-rate in acceleration system. 
The inertial force is expressed as:

$$
F_{k}=\frac{\mathrm{d} E_{k}}{\mathrm{~d} r}=\frac{1}{\mathscr{F}^{3}} m_{0} a
$$

But according to the relativistic dynamics, the inertial force was determined by the Equation below in motorial system with variable speed:

$$
F=\frac{\mathrm{d}}{\mathrm{d} t}(m u)=m \frac{\mathrm{d} u}{\mathrm{~d} t}+u \frac{\mathrm{d} m}{\mathrm{~d} t}
$$

Substituting the $m=\frac{m_{0}}{\mathscr{F}}$ into Equation above, then:

$$
F=\frac{m_{0}}{\mathscr{F}} \frac{\mathrm{d} u}{\mathrm{~d} t}+m_{0} u \frac{\mathrm{d} \mathscr{F}^{-1}}{\mathrm{~d} t}=m_{0} \frac{1}{\mathscr{F}^{3}} \frac{\mathrm{d} u}{\mathrm{~d} t}
$$

And this is exactly what the Equation (32) above.

\section{Conclusions}

According to the same principle, we have come to the conclusion that gravitation, centrifugal force, and inertial force could be unified with the idea of gradient of time-rate. Through the study in the above chapter, there is no essential difference for function mechanism between inertia force and gravitation. On the contrary, it forms gradient of time-rate by a certain way in the region of space, and effects on the mass through it. In a sense, the inertia force is a kind of manifestation of gravitation. So, the gravitation and the inertia force are equivalent.

Usually, the external force must be applied to an object passively and makes it speed up, but it will be dragged by inertia force in the opposite direction of acceleration, which makes acceleration become much more difficult because of inertia.

However, if there is no external force applied to an object factitiously, but to form gradient of time-rate in a Specific direction in interior region of the object, then it will attain the acceleration by the physical field acts; at the same time, there would form gradient of time-rate in the opposite direction because of the inertia force effect to counteract the force. So no matter how great the acceleration of the object has, or how it moves with variable speed in motion whatever, you couldn't be alive to any inertial force effects inside the object. It was just similar to the body in the state of free fall in gravitation field. Therefore, because of the superposition of these two effects mutually, thus, an ideal physical model is established whose magnitude and direction of gradient of time-rate can be changed, and make the object get huge acceleration instantly, but internal space always maintains balance.

\section{Acknowledgements}

Upon the completion of the manuscript, I greatly appreciate the assistance offered by the authors in the references, without whose works, the paper would not have been possible. 


\section{Conflicts of Interest}

The author declares no conflicts of interest regarding the publication of this paper.

\section{References}

[1] Cai, B.L. (1992) Special Theory of Relativity. Higher Education Publisher, Beijing, 39.

[2] Wu, D.Y. (1983) Theory of Relativity-Theories Physics Volume 4. Science Publisher, Beijing, 170. 